\title{
PERIODONTITIS AND RHEUMATOID ARTHRITIS: A CASE-CONTROL STUDY
}

Nelzi Ferreira de Queiroz Junior ${ }^{1, \star}$, Laís Zanlorenzi ${ }^{1}$, Angelo Cesar Crisigiovanni ${ }^{1}$, Renato Nishiara ${ }^{1}$, Allan Fernando Giovanini ${ }^{1}$, Barbara Stadler Kahlow ${ }^{1}$, Thelma Larocca Skare ${ }^{1}$

1. Hospital Universitário Evangélico Mackenzie, Curitiba (PR), Brazil.

*Corresponding author: nelzi@hotmail.com

\section{BACKGROUND}

Periodontitis and rheumatoid arthritis (RA) may be linked to each other. In both diseases, hard and soft tissue destruction is seen with pro inflammatory cytokines such as IL (interleukin)-6, TNF-alpha and IL-1 playing a role. Several studies have detected increased periodontal disease in RA patients and periodontitis is considered to influence in the RA appearance and in the disease activity. The aim of this work was to study the oral health of RA patients comparing it with controls in a Brazilian sample from a public health rheumatologic center.

\section{METHODS}

One hundred and sixteen RA patients and 68 controls (patients' companions) were compared for epidemiological data and presence of periodontal disease evaluated by number of remaining teeth, presence of bacterial plaque, bleeding on probing, probing depth and clinical level of gingival insertion. Periodontitis was classified in severe, moderate and mild. Data on comorbidities was also collected

\section{RESULTS}

Patients and controls were paired for gender, age, tobacco use, years of formal education and presence of diabetes mellitus, hypothyroidism, hypertension and dyslipidemia. Rheumatoid arthritis patients and controls have the same amount of teeth loss/ person (11 vs. 9; $p=0.84)$; RA patients had more calculus ( $p=0.02)$; dental plaques $(p=0.04)$; gingival retraction $(p=0.02)$ and bleeding ( $p=0.01$ ). Although the number of individuals with periodontitis was higher in RA patients ( $56.1 \% \mathrm{vs} .32 .8 \%$ ), the severity of periodontitis was similar in both groups $(p=n s)$. Diabetes and hypothyroidism also associated with periodontitis $(p=0.01$ and 0.02 , respectively). In a model of logistic regression built to assess the independence of association of RA and its comorbidities with periodontitis, only diabetes $(p=0.006 ; O R=5.2 ; 95 \% C l=1.5-17.1)$ and $R A(p=0.01 ; O R=2.45 ; 95 \% C l=1.1-5.2)$ remained independent.

\section{CONCLUSION}

This case control study shows that oral health in RA patients is poorer than controls. Diabetes mellitus also associated with periodontitis.

\section{KEYWORDS}

Rheumatoid arthritis, Periodontitis, Tooth. 